\title{
RESENAS BIBLIOGRÁFICAS
}

\section{RESEÑA DE "ESTUDIOS SOBRE LA MUJER TRABAJADORA Y SU PROTECCIÓN JURÍDICA"}

(GÓMEZ SALADO, M.Á. (DIRECTOR); ARAGÜEZ VALENZUELA, L. D, MACÍAS GARCÍA, M. C., ORDOÑEZ CASADO, M.I., RUIZ SANTAMARÍA, J.L. Y VIGO SERRALVO, F. (COORDINADORES), EDICIONES LABORUM, MURCIA, 2019)*

\section{Jorge Baquero Aguilar}

Profesor de Derecho del Trabajo y de la Seguridad Social

Universidad de Málaga

jbaquero@uma.es ORCID 0000-0002-8524-7947

Recepción de trabajo: 11-08-2020-Aceptación: 13-08-2020

La evolución de los derechos de la mujer ha sufrido un cambio muy considerable y a la vez importante dentro de nuestra sociedad a lo largo de las últimas décadas en nuestro país. Aparejado a los mismos; afortunadamente, han ido evolucionando también los derechos laborales de las mismas. En ese sentido, esta obra que venimos reseñando pretende analizar dichos derechos en los tiempos que corren desde un punto de vista ecléctico y multidisciplinar.

Profesores de diversas Universidades, Ayudantes Doctores, Contratados Predoctorales, Profesores Visitantes, Colaboradores Honorarios y Graduados Sociales, Funcionarios y Abogados Doctorandos de otros países, todos ellos pertenecientes al ámbito del Derecho del Trabajo y de la Seguridad Social; así como también Graduados en Derecho y Dirección y Administración de Empresas, Investigadores y Profesores del ámbito del Trabajo Social y de los Servicios Sociales, Profesores de Derecho Financiero y Tributario; investigadores consolidados y nóveles de diferentes disciplinas, procuran un tratamiento oportuno y eficaz desde diferentes puntos de vista sobre la protección jurídica que brinda nuestro ordenamiento jurídico a la mujer trabajadora en nuestra sociedad actual; desde un punto de vista tanto del ordenamiento jurídico nacional como desde el derecho internacional.

* $\quad$ Este trabajo resen̂ado se inserta dentro de las actividades del Grupo de Investigación PAIDI SEJ-347 sobre "Políticas de empleo, igualdad e inclusión social", financiado por la Junta de Andalucía. 
"ESTUDIOS SOBRE LA MUJER TRABAJADORA Y SU PROTECCIÓN JURÍDICA"

Como venimos anunciando, esta obra colectiva' pretende llevar a cabo un análisis de la situación actual de la mujer trabajadora y de su protección jurídica desde diferentes perspectivas. Se estructura la misma en diecisiete capítulos que nos otorgan diferentes perspectivas jurídicas de dicha protección tanto desde un punto de vista nacional como internacional, así como desde la perspectiva del derecho comparado.

A modo de introducción, en el Capítulo 0 el profesor Francisco Vila Tierno; además de hacer una presentación de la obra y de sus autores, lleva a cabo un planteamiento de la cuestión en relación a la "Mujer y Trabajo" realizando un análisis de la normativa tanto internacional y comunitaria como nacional al respecto; prestando especial atención a las medidas relacionadas con la maternidad en nuestro país.

En el Capitulo I, el profesor Salvador Perán Quesada aborda "La protección de la trabajadora embarazada frente al despido" desde el punto de vista de la integración de la perspectiva de género en su amplio sentido del concepto en la normativa laboral; así como también analiza la evolución normativa y jurisprudencial de dicha protección a lo largo de los años en nuestro país (tanto a nivel nacional como internacional); terminando su análisis con una disertación sobre "La particular tutela de la trabajadora embarazada ante el libre desistimiento durante el período de prueba" derivado de los últimas resoluciones jurisprudenciales al respecto; poniendo de manifiesto la no todavía consolidación definitiva de la protección y tutela de los derechos de la mujer trabajadora que se encuentra en situación de embarazo.

Por su parte, el profesor Miguel Ángel Gómez Salado, en el Capítulo II lleva a cabo un análisis de "La extinción objetiva del contrato de trabajo por faltas de asistencia y la protección de las mujeres trabajadoras víctimas de la violencia de género"; realizando un análisis exhaustivo tanto desde el punto de vista legal como convencional de esta situación donde se pone de manifiesto los vacíos legales al respecto de esta cuestión tratada.

La perspectiva de derecho comparado la introduce en el Capítulo III la profesora Shelagh Anne Mckenzie con su trabajo "Working women in the United Kingdom: an overview comparative perspective"; donde se realiza un análisis comparado de la protección de los derechos de la mujer en relación al ordenamiento jurídico español y del Reino Unido: embarazo y maternidad, licencia parental no remunerada, licencia por incumplimiento, trabajo flexible, brecha salarial, acoso sexual, mujeres en puestos de responsabilidad, etc.

La profesora Lucía Dolores Aragüez Valenzuela analiza "El papel de la mujer trabajadora en los contratos de cero horas en Reino Unido y su impacto en la "Cig Economy" en el Capítulo IV de esta obra; poniendo en consideración la importante relación entre el tiempo de trabajo, este tipo de contratos y la precarización laboral; así como llevando a cabo también una comparación entre hombres y

1 Obra que es fruto de las intervenciones de todos estos investigadores en el "I Congreso Internacional sobre la Protección Jurídica de la Mujer Trabajadora: el camino hacia la Igualdad", celebrado en Málaga en el 29 de junio de 2018. 
"ESTUDIOS SOBRE LA MUJER TRABAJADORA Y SU PROTECCIÓN JURÍDICA"

mujeres (estas últimas sufren en mayor medida este tipo de contratación); así como poniendo de relieve el impacto de estas nuevas formas de trabajo en la "Gig Economy" derivado del fuerte crecimiento de las nuevas tecnologías relacionadas con el trabajo; provocando una mayor flexibilidad del mismo que conlleva una desregulación de las RRLL.

En el Capítulo V, el profesor Francisco Miguel Ortiz González-Conde, partiendo de una serie de sentencias de diferentes instancias, analiza la "Discriminación Laboral a causa de la maternidad y el embarazo"; afrontando la problemática de las dificultades en el ejercicio de la conciliación laboral, familiar y personal, el bloqueo de los derechos salariales y profesionales, los despidos como móvil discriminatorio hacia la mujer, la discriminación que se produce en el período de prueba y las discriminaciones producidas en la contratación laboral.

El profesor José Luis Ruiz Santamaría, en el Capítulo VI nos procura una serie de interesantes "Reflexiones jurídicas sobre el alcance del 'deber' de comunicación en relación a la protección de la trabajadora embarazada, en posparto y lactancia"; Ilevando a cabo un análisis normativo de la legislación nacional e internacional -incluso con matices de Derecho comparado-, y analizando las principales resoluciones jurisprudenciales al respecto. Examinando además el deber de comunicación de la mujer trabajadora así como las medidas preventivas y de protección de la misma y la tutela de sus derechos contra la discriminación en el empleo.

"La configuración jurídico-preventiva del riesgo del embarazo" es el tema elegido por parte de la profesora María del Carmen Macías García en el Capítulo VII. Se revisa en este trabajo la configuración jurídico-histórica de la protección de la mujer y la maternidad, el riesgo del embarazo en un sentido amplio (situación de embarazo, riesgo, existencia del riesgo conforme al criterio jurisprudencial, el procedimiento de solicitud) y los riesgos laborales en el embarazo.

María de Monserrate Rodríguez Egío lleva a cabo una interesante análisis de "La protección del embarazo en el trabajo autónomo" en el Capítulo VIII; donde se analiza la situación especial y la problemática concreta de las trabajadoras autónomas ante los riesgos que pueden plantearse en el embarazo y la lactancia natural, poniendo de manifiesto las diferencias legales suscitadas con respecto a una trabajadora por cuenta ajena; así como todas las vicisitudes y problemas que plantea esta situación para una trabajadora por cuenta propia; pudiéndose plantear, incluso, situaciones de discriminación con respecto a una trabajadora por cuenta ajena.

Desde un punto de vista más práctico, Virginia Cárdeñas Porta, disecciona en el Capítulo IX “La tutela de la mujer en la acción protectora de la Seguridad Social. Especial referencia al subsidio contributivo de maternidad"; aportando en su trabajo la experiencia de la práctica profesional derivada de los años de ejercicio como Graduada Social.

El Capítulo X, elaborado por parte de la profesora Patricia Espejo Megías, versa sobre "La pensión de viudedad en la actualidad. Especial atención a las parejas de hecho y a las mujeres víctimas de violencia de género". Se realiza un interesantísimo análisis del recorrido normativo a lo largo de los años en nuestro país; así como el controvertido encaje constitucional de los requisitos exigidos a las parejas de hecho y la situación especial de las mujeres víctimas de violencia de género. 
"ESTUDIOS SOBRE LA MUJER TRABAJADORA Y SU PROTECCIÓN JURÍDICA"

El profesor Juan Carlos Álvarez Cortés nos otorga un oportuno, detallado y acertado análisis y estudio de las "Bonificaciones y reducciones como incentivos a la contratación de las mujeres" en el Capítulo XI de esta obra. Examina los datos sobre el colectivo femenino en relación al desempleo y a las políticas de empleo, la reducción de cotizaciones, los incentivos a la contratación a través de las bonificaciones y reducciones de las cuotas de la Seguridad Social tanto en el trabajo por cuenta ajena como por cuenta propia; así como la eficacia de dichas bonificaciones; planteando un reenfoque en las bonificaciones y reducciones para el colectivo de mujeres.

Las políticas de empleo y su relación con el género son tratadas por parte de la profesora María Iluminada Ordóñez Casado de una forma muy pertinente y minuciosa en el Capítulo XII: "Un recorrido histórico por las normas internacionales, europeas y estatales sobre las políticas de empleo desde la perspectiva de género"; donde se presta especial atención al principio de igualdad y la prohibición de discriminación en el ordenamiento jurídico español.

Por su parte, el profesor Francisco Vigo Serralvo, en el Capítulo XIII, reflexiona sobre "La normativa laboral de fomento de la paridad entre sexos". Parte de una diferenciación de Derecho del Trabajo y del Derecho del Empleo, centrando su atención en la paridad en el acceso al empleo analizando tanto la normativa nacional como internacional referente y sus respectivas vicisitudes; resaltando la importancia de la Ley Orgánica 3/2007 en relación a la paridad de sexos y el acceso al empleo, la proscripción de criterios discriminatorios en los procesos selectivos y de reclutamiento de personal, las políticas de empleo y su desarrollo, el distintivo para las empresas en materia de igualdad, la importancia de la negociación colectiva al respecto de la cuestión (con especial atención a los planes de igualdad) y los tipos infractores en materia de igualdad en el acceso al empleo.

El aspecto social en términos de la disciplina del Trabajo Social lo introducen las "Medidas para impulsar el empleo de las mujeres en riesgo de exclusión social", que son analizadas por parte del profesor Mario Millán Franco, la Graduada en Derecho y Dirección y Administración de Empresas Sofía Louise Martínez Martínez y la profesora Laura Domínguez de la Rosa en el Capítulo XIV de esta obra. Se analizan en este capítulo los indicadores y la conceptualización de la exclusión social, la necesidad del enfoque de género en el fenómeno de la exclusión social haciendo especial hincapié en los colectivos de mujeres con alto riesgo de exclusión social; así como en las medidas para impulsar el empleo de calidad de las mujeres en riesgo de exclusión social en aras de una búsqueda de la plena inclusión laboral de las mismas.

El punto de vista del análisis económico internacional de la cuestión es introducido por parte de Ios Doctorandos Manoela Papp (Funcionaria Pública de Justicia en Brasil) y Luis Felipe Seegmuller de Carvalho (Abogado en Brasil) en el Capítulo XV con su trabajo "La necesidad del equilibrio de géneros en los mercados financieros". Para estos autores, es en el mercado financiero donde se produce una mayor distorsión de representación entre géneros; a pesar de que existen estudios que demuestran mejor rendimiento que el obtenido por los hombres; lo que analizan de una forma detallada a lo largo de su trabajo. 
Por último, y desde el punto de vista fiscal, en el Capítulo XVI, el profesor Miguel Gutiérrez Bengoechea analiza el "Género y la fiscalidad en el régimen matrimonial de gananciales". Se revisan los derechos y obligaciones de los distintos regímenes matrimoniales, las cuestiones tributarias en el IRPF y otro tipo de impuestos en gananciales; así como las modificaciones de lege ferenda en la fiscalidad del régimen económico ganancial.

En definitiva; y tal y como podrá observar el lector de esta obra colectiva, en la misma se lleva a cabo un exhaustivo a análisis de la situación jurídica y social en su amplio sentido de la mujer trabajadora y de los mecanismos para su protección y tutela desde diferentes puntos de vista y desde diferentes disciplinas (Derecho del Trabajo, Derecho de la Seguridad Social, Derecho del Empleo, Prevención de Riesgos Laborales, Derecho a la Igualdad y Derecho Constitucional; Derecho Tributario, notas de Derecho comparado y de la perspectiva del ámbito económico y del Trabajo Social).

Todo ello no hace sino encumbrar esta obra ecléctica y multidisciplinar como obra de referencia dentro del estudio de la protección de los derechos de la mujer trabajadora inserta en nuestro panorama actual. Obra que se presenta como necesaria tanto por su calidad como por la profundidad y diversificación de los temas tratados; así como por las diferentes perspectivas desde las que se aborda la cuestión de la protección jurídica de la mujer trabajadora en nuestro país. 